Pacific Journal of Mathematics

ON EXTREME POINTS AND SUPPORT POINTS OF THE
FAMILY OF STARLIKE FUNCTIONS OF ORDER $\alpha$ 


\title{
ON EXTREME POINTS AND SUPPORT POINTS OF THE FAMILY OF STARLIKE FUNCTIONS OF ORDER $\alpha$
}

\author{
A. A. S. Perera and D. R. Wilken
}

Let $\operatorname{St}(\alpha)$ denote the subclass of functions $f(z)$ analytic in the open unit disk $D$ which satisfy the conditions $f(0)=0, f^{\prime}(0)=1$ and $\operatorname{Re}\left(z f^{\prime}(z) / f(z)\right)>\boldsymbol{\alpha}$ for $z$ in $D$. In this note we investigate the compact, convex family $\operatorname{co} S(\operatorname{St}(\alpha))$ which is the closed convex hull of the set of all functions analytic in $D$ that are subordinate to some function in $\operatorname{St}(\alpha)$, $\alpha<1 / 2$. The principal result establishes that every support point of $\operatorname{co} S(\operatorname{St}(\alpha))$ arising from a "nontrivial" functional must also be an extreme point, hence a function of the form $f(z)=x z /(1-y z)^{2(1-\alpha)}$, $|x|=|y|=1$.

To amplify on this synopsis, let $\mathscr{A}$ denote the set of functions analytic in the open unit disk $D=\{z \in \mathrm{C}|| z \mid<1\}$. Then $\mathscr{A}$ is a locally convex linear topological space under the topology of uniform convergence on compact subsets of $D$. A function $f$ in $\mathscr{A}$ is said to be subordinate to a function $F$ in $\mathscr{A}$ (written $f \prec F$ ), if there is a function $\varphi$ in $B_{0}$ such that $f(z)=F(\varphi(z))$, where $B_{0}=\{\varphi \in \mathscr{A} \mid \varphi(0)=0$, $|\varphi(z)|<1$ in $D\}$.

Let $\mathscr{F}$ be a compact subset of $\mathscr{A}$. A function $f$ in $\mathscr{F}$ is a support point of $\mathscr{F}$ if there is a continuous linear functional $J$ on $\mathscr{A}$ such that

$$
\operatorname{Re} J(f)=\max \{\operatorname{Re} J(g) \mid g \in \mathscr{F}\}
$$

and $\operatorname{Re} J$ is non-constant on $\mathscr{F}$. We use $\Sigma \mathscr{F}$ to denote the set of support points of $\mathscr{F}$ and $\overline{\operatorname{co}} \mathscr{F}$ and $\mathscr{E} \overline{c o} \mathscr{F}$ to denote, respectively, the closed convex hull of $\mathscr{F}$ and the set of extreme points of the closed convex hull of $\mathscr{F}$.

Let $S(\operatorname{St}(\alpha))$ denote the set of functions in $\mathscr{A}$ that are subordinate to some function in $\operatorname{St}(\alpha)$. Then $S(\operatorname{St}(\alpha))$ is a compact subset of $\mathscr{A}[11, \mathrm{p}$. 365]. In [3] and [6] it was shown that

$$
\overline{\operatorname{cost}}(\alpha)=\left\{\int \frac{z}{(1-x z)^{2(1-\alpha)}} d \mu(x): \mu\right. \text { is a probability measure }
$$

the unit circle

and that

$$
\mathscr{E} \operatorname{cost}(\alpha)=\sum \operatorname{St}(\alpha)=\left\{\frac{z}{(1-x z)^{2(1-\alpha)}}:|x|=1\right\} .
$$


The analogous questions for $S(\operatorname{St}(\alpha))$ have not been so readily answered and only recently has a reasonably complete description been presented. Hallenbeck [8] and Hallenbeck and MacGregor [9] obtained $\operatorname{co} S(\operatorname{St}(\alpha))$ for $\alpha \leq 0$ and $\alpha=1 / 2$ in 1974. The missing link, $0<\alpha<1 / 2$, was completed by Perera in his doctoral dissertation [12]. Thus we now have

TheOREM (Hallenbeck, MacGregor, Perera). Let $\alpha \leq 1 / 2$. Then

$$
\overline{\operatorname{co}} S(\operatorname{St}(\alpha))=\left\{\int \frac{x z}{(1-z y)^{2(1-\alpha)}} d \mu(x, y): \mu\right. \text { is a probability }
$$

measure on the torus $\}$,

$$
\mathscr{E} \overline{\cos } S(\operatorname{St}(\alpha))=\left\{\frac{x z}{(1-y z)^{2(1-\alpha)}}:|x|=|y|=1\right\} .
$$

If $1 / 2<\alpha<1$ and $p=2(1-\alpha)$, then $0<p<1$ and the usual arguments break down. One encounters difficulties analogous to those for the families $V_{k}$ of functions with bounded boundary rotation, when $2<k<4$, or the families $C(\beta)$ of close-to-convex functions of order $\beta$, when $0<\beta<1$.

Also in [3] the following sharp inequalities were obtained (for $\alpha=0$ see [13]): If $f$ is in $S(\operatorname{St}(\alpha))$ and $f(z)=\sum_{n=1}^{\infty} a_{n} z^{n}$, then, for

$$
\alpha \leq 0, \quad\left|a_{n}\right| \leq \frac{(2-2 \alpha)(3-2 \alpha) \cdots(n-2 \alpha)}{(n-1) !} \quad(n=1,2, \ldots)
$$

and, for $1 / 2 \leq \alpha<1,\left|a_{n}\right| \leq 1(n=1,2, \ldots)$.

In [12] Perera also obtains, for $\alpha \leq 1 / 2$, the support points of $\overline{\operatorname{co}} S(\operatorname{St}(\alpha))$ as a consequence of a somewhat more general result. In this note we show that the first inequality above for the coefficients also obtains in the range $0<\alpha<1 / 2$, and examine the support points of $S(\operatorname{St}(\alpha))$ for $\alpha<1 / 2$. In [10] Hallenbeck and MacGregor discussed the case $\alpha=0$ and we extend this by showing, for $\alpha<1 / 2$, that if $f$ is a support point of $S(\operatorname{St}(\alpha))$ corresponding to a continuous linear functional $J$ on $\mathscr{A}$ not of the form $J(f)=a f(0)+b f^{\prime}(0)(f \in \mathscr{A}, a, b \in \mathbf{C})$, then $f$ is an extreme point of $\overline{\operatorname{co}} S(\operatorname{St}(\alpha))$. 
1. Extreme points of the closed convex hull of $S(\operatorname{St}(\alpha))(\alpha \leq 1 / 2)$.

LEMMA 1.1. Let $U$ denote the unit circle $\{z \in \mathbf{C}|| z \mid=1\}$ and let $\mu$ and $\nu$ be two probability measures on $U$. If $p$ and $q$ are two non-negative real numbers with $p+q \geq 1$, then there exists a probability measure $\lambda$ on $U \times U$ such that

$$
\begin{gathered}
\left\{\int_{U} \frac{x z}{(1-x z)^{p}} d \mu(x)\right\}\left\{\int_{U} \frac{1}{(1-y z)} d \nu(y)\right\}^{q} \\
=\int_{U \times U} \frac{x z}{(1-y z)^{p+q}} d \lambda(x, y) .
\end{gathered}
$$

Proof. It is well known that $\log (1-z)$ is univalent and convex. It follows that, if $f(z) \prec 1 /(1-z)^{p}$ and $g(z) \prec 1 /(1-z)^{q}$, then

$$
f(z) \cdot g(z) \prec \frac{1}{(1-z)^{p+q}} .
$$

This fact together with a trivial modification of the Herglotz formula yields

$$
\frac{1}{(1-x z)^{p}} \cdot\left\{\int_{U} \frac{1}{(1-y z)} d \nu(y)\right\}^{q} \prec \frac{1}{(1-z)^{p+q}} .
$$

Since $p+q \geq 1$ a result of Brannan, Clunie and Kirwan ([2], p. 5) yields

$$
\frac{1}{(1-x z)^{p}} \cdot\left\{\int_{U} \frac{1}{(1-y z)} d \nu(y)\right\}^{q}=\int_{U} \frac{1}{(1-w z)^{p+q}} d \alpha(w),
$$

for some probability measure $\alpha$ on $U$. Hence we have

$$
\begin{gathered}
\left\{\int_{U} \frac{x z}{(1-x z)^{p}} d \mu(x)\right\}\left\{\int_{U} \frac{1}{(1-y z)} d \nu(y)\right\}^{q} \\
=\int_{U \times U} \frac{x z}{(1-w z)^{p+q}} d \alpha(w) d \mu(x) .
\end{gathered}
$$

Now it is easy to see that the right hand side of the above equation belongs to the set

$$
\left\{\int_{U \times U} \frac{x z}{(1-y z)^{p+q}} d \lambda(x, y) \mid \lambda \text { is a probability measure on } U \times U\right\}
$$

and the lemma follows. 
TheOREM 1.2. Let $U$ be the unit circle $\{z \in \mathbf{C}|| z \mid=1\}$ and $\alpha \leq 1 / 2$. Also let $\mathscr{F}$ consist of the functions

$$
f_{\lambda}(z)=\int_{U \times U} \frac{x z}{(1-y z)^{2(1-\alpha)}} d \lambda(x, y),
$$

where $\lambda$ varies over the probability measures on $U \times U$. Then $\overline{\operatorname{co}} S(\operatorname{St}(\alpha))=$ $\mathscr{F}$ and

$$
\mathscr{E} \operatorname{co} S(\operatorname{St}(\alpha))=\left\{\frac{x z}{(1-y z)^{2(1-\alpha)}}|| x|=| y \mid=1\right\} .
$$

Proof. This theorem was known for $\alpha \leq 0$ and $\alpha=1 / 2$ ([9], [8]). Our aim here is to prove it for $0<\alpha<1 / 2$. The main tool is Lemma 1.1.

Suppose that $f$ is in $\mathscr{E} \operatorname{co} S(\operatorname{St}(\alpha))$. Then a result in [11, p. 366] implies that $f \prec g$ for some $g \in \mathscr{E} \overline{\operatorname{co}} \operatorname{St}(\alpha)$. $\mathscr{E} \operatorname{coSt}(\alpha))$ was found in [3, p. 417] to be the set of all functions

$$
\frac{z}{(1-x z)^{2(1-\alpha)}} \text { with }|x|=1
$$

Hence we have

$$
f(z)=\frac{\varphi(z)}{(1-c \varphi(z))^{2(1-\alpha)}}
$$

for some $|c|=1$ and $\varphi$ in $B_{0}$. Write $f(z)$ in the form

$$
f(z)=\bar{c}\left\{\frac{c \varphi(z)}{1-c \varphi(z)}\right\} \cdot\left\{\frac{1}{1-c \varphi(z)}\right\}^{(1-2 \alpha)}
$$

First using trivial modifications of the Herglotz formula and then applying the Lemma 1.1 with $p=1$ and $q=1-2 \alpha(q \geq 0$ if $\alpha \leq 1 / 2)$ we obtain

$$
f(z)=\bar{c} \int_{U \times U} \frac{x z}{(1-y z)^{2(1-\alpha)}} d \lambda(x, y)
$$

for some probability measure $\lambda$ on $U \times U$. Since

$$
\frac{\bar{c} x z}{(1-y z)^{2(1-\alpha)}} \in \mathscr{F}, \quad \text { for all }|c|=|x|=|y|=1,
$$

and $\mathscr{F}$ is compact and convex, it is clear that $f \in \mathscr{F}$. Hence $\mathscr{E} \operatorname{co} S(\operatorname{St}(\alpha))$ $\subseteq \mathscr{F}$ and $\overline{\operatorname{co}} S(\operatorname{St}(\alpha)) \subseteq \mathscr{F}$. On the other hand

$$
\frac{x z}{(1-y z)^{2(1-\alpha)}} \in S(\operatorname{St}(\alpha))
$$


which implies that $\mathscr{F} \subseteq \overline{\operatorname{co}} S(\operatorname{St}(\alpha))$ and $\overline{\operatorname{co}} S(\operatorname{St}(\alpha))=\mathscr{F}$. Now Theorem 1.1 in [4] yields

$$
\mathscr{E} \overline{\operatorname{co}} S(\operatorname{St}(\alpha)) \subseteq\left\{\frac{x z}{(1-y z)^{2(1-\alpha)}}|| x|=| y \mid=1\right\} .
$$

These sets are actually equal. For, if

$$
\frac{x_{0} z}{\left(1-y_{0} z\right)^{2(1-\alpha)}}=\int_{U \times U} \frac{x z}{(1-y z)^{2(1-\alpha)}} d \lambda(x, y),
$$

then by now standard methods we obtain $x_{0}=\int_{U \times\left\{y_{0}\right\}} x d \lambda(x, y)$ and $\lambda\left(\left\{x_{0}, y_{0}\right\}\right)=1$. Hence the theorem.

COROLlaRY 1.3. Let $f(z) \in S(\operatorname{St}(\alpha))$ and $f(z)=\sum_{n=1}^{\infty} a_{n} z^{n}$. If $\alpha \leq$ $1 / 2$, then

$$
\left|a_{n}\right| \leq \frac{(2-2 \alpha)(3-2 \alpha) \cdots(n-2 \alpha)}{(n-1) !} \quad(n=1,2, \ldots)
$$

and the inequality is sharp.

Proof. This follows immediately from Theorem 1.2 and the argument given in [11, p. 366].

Remarks. (1) Corollary 1.3 was known for $\alpha=0$, a result of W. Rogosinski [13, p. 72] and for $\alpha \leq 0$ and for $\alpha=1 / 2$ [8, p. 61]. Since the sharp bounds for the Taylor coefficients were also known for $1 / 2 \leq \alpha<1$ [3, p. 423], we have now completed the determination of sharp bounds for the Taylor coefficients of the functions in $S(\operatorname{St}(\alpha))$.

(2) It was noted in [8] that Theorem 1.2 is not true for $1 / 2<\alpha<1$. We note that if $1 / 2<\alpha<1$ then $\overline{\operatorname{co}} S(\operatorname{St}(\alpha))$ has a large number of extreme points. We claim that if $1 / 2<\alpha<1$, then

$$
\psi(z) /(1-x \psi(z))^{2(1-\alpha)}
$$

belongs to $\mathscr{E} \overline{\operatorname{co}} S(\operatorname{St}(\alpha))$ where $\psi(z)$ is an inner function with $\psi(0)=0$ and $|x|=1$. For, if

$$
\frac{\psi(z)}{(1-x \psi(z))^{2(1-\alpha)}}=t f_{1}(z)+(1-t) f_{2}(z),
$$

where $0<t<1$ and $f_{1}(z), f_{2}(z) \in \mathscr{E} \operatorname{co} S(\operatorname{St}(\alpha))$, then

$$
\frac{z}{(1-x z)^{2(1-\alpha)}} \in H^{q}
$$


for some $q>1($ since $1 / 2<\alpha<1)$ and

$$
\left\|f_{1}\right\|_{q},\left\|f_{2}\right\|_{q} \leq\left\|\frac{z}{(1-z)^{2(1-\alpha)}}\right\|_{q} .
$$

The conclusion that $f_{1}(z)=f_{2}(z)$ can be drawn exactly the same way as in $[9$, p. 466]. Hence the claim.

2. Support points of a family related to $S(\operatorname{St}(\alpha))$. Let $U$ be the unit circle and

$\mathscr{G}_{p}=\left\{\int_{U \times U} \frac{x z}{(1-y z)^{p}} d \mu(x, y) \mid\right.$

$$
\mu \text { is a probability measure on } U \times U\} \quad(p>0) .
$$

In $\S 1$ we showed that, if $\alpha \leq 1 / 2$, then $\mathscr{G}_{2(1-\alpha)}=\overline{\operatorname{co}} S(\operatorname{St}(\alpha))$. In this section we are interested in determining the support points of the compact convex family $\mathscr{G}_{p}$. In $\S 3$ we use this result when we consider the problem of support points of $S(\operatorname{St}(\alpha))$. We first need a theorem from the first named author's doctoral dissertation and a lemma. We reproduce the proof of the theorem for completeness.

Lemma 2.1. ( D. Cantor, R. R. Phelps [5].) Let $a_{1}, \ldots, a_{n}$ be complex numbers with $\left|a_{k}\right|=1(k=1,2, \ldots, n)$ and $b_{1}, \ldots, b_{n}$ be distinct complex numbers with $\left|b_{k}\right|=1(k=1,2, \ldots, n)$. Then there exists a finite Blaschke product $B(z)$ such that $B\left(b_{k}\right)=a_{k}(k=1,2, \ldots, n)$.

THEOREM 2.2.

$$
\sum \mathscr{G}_{p}=\left\{\int_{U} \frac{\overline{B(y)} z}{(1-y z)^{p}} d \mu(y) \mid B\right. \text { is a finite }
$$

Blaschke product and $\nu$ is a probability measure on $U\}$.

Proof. First note that

$$
\mathscr{E} \mathscr{G}_{p}=\left\{\frac{x z}{(1-y z)^{p}}|| x|=| y \mid=1\right\} .
$$


We begin as in [7]. Suppose that $f$ is a support point of $\mathscr{G}_{p}$. Then there is a continuous linear functional $J$ on $\mathscr{A}$ such that $\operatorname{Re} J(f)=$ $\max \left\{\operatorname{Re} J(g) \mid g \in \mathscr{G}_{p}\right\}$ and $\operatorname{Re} J$ is non constant on $\mathscr{G}_{p}$. If we let $M=$ $\max \left\{\operatorname{Re} J(g) \mid g \in \mathscr{E} \mathscr{G}_{p}\right\}$, then the above equation becomes $\operatorname{Re} J(f)=M$, and

$$
f(z)=\int_{U \times U} \frac{x z}{(1-y z)^{p}} d \mu(x, y),
$$

for some probability measure $\mu$ on $U \times U$. Hence we have

$$
\operatorname{Re} J\left\{\frac{x z}{(1-y z)^{p}}\right\}=M
$$

$\mu$ a.e. on $U \times U$, i.e. $\operatorname{Re} x F(y)=M, \mu$ a.e. on $U \times U$, where $F(y)=$ $J\left\{z /(1-y z)^{p}\right\}$ is analytic in $\bar{D}$. If $\operatorname{Re} x f(y)=M$ holds at $\left(x_{1}, y_{1}\right)$ then $F\left(y_{1}\right) \neq 0$, for otherwise $M=0$ and it follows that $J$ is constant on $\mathscr{G}_{p}$. Thus $|f(y)|=M, \mu$ a.e. on $U \times U$, and $x$ is uniquely determined by $x F(y)=|F(y)|$.

Case (i). $|F(y)|=M$ holds only for finitely many values of $y$.

Then

$$
f(z)=\sum_{k=1}^{n} \lambda_{k} \frac{x_{k} z}{\left(1-y_{k} z\right)^{p}} \quad \text { where }\left|x_{k}\right|=1=\left|y_{k}\right|, \quad \lambda_{k}>0,
$$

$$
(k=1,2, \ldots, n)
$$

and $\sum_{k=1}^{n} \lambda_{k}=1$.

Case (ii). $|F(y)|=M$ holds for infinitely many values of $y$.

Then, as in [10, p. 539], $F(y)=M B(y)$ for some finite Blaschke product $B(z), x$ is determined by $x B(y)=1$ and the support of $\mu$ is the set $T=\{(x, y) \in U \times U \mid x B(y)=1\}$. Then

$$
f(z)=\int_{T} \frac{\bar{B}(y) z}{(1-y z)^{p}} d \mu(x, y) .
$$

Now for any Borel set $A$ of $U$ define $\nu(A)=\mu(C)$ where $C(\subseteq T)$ is the image of $A$ under the homeomorphism $y \rightarrow(\overline{B(y)}, y)$ of $U$ onto $T$. Clearly $\nu$ is a probability measure and $f(z)$ takes the form

$$
f(z)=\int_{U} \frac{\overline{B(y)} z}{(1-y z)^{p}} d \nu(y) .
$$

The form for $f(z)$, obtained in case (i), can also be written in the above form. For we can use Lemma 2.1 with $b_{k}=y_{k}$ and $a_{k}=\bar{x}_{k}$. 
Conversely

$$
\int_{U} \frac{\overline{B(y)} z}{(1-y z)^{p}} d \nu(y)
$$

is a support point of $\mathscr{G}_{p}$ for each finite Blaschke product $B(z)$ and for each probability measure $\nu$ on $U$. To see this choose a continuous linear functional $J$ on $\mathscr{A}$ such that $J\left\{z /(1-y z)^{p}\right\}=B(y)$. (This is easily seen to be possible.) It is immediate that $\operatorname{Re} J$ is non constant and peaks at

$$
\int \frac{\overline{B(y)} z}{(1-y z)^{p}} d \nu(y) \text {. }
$$

\section{Support points of $S(\operatorname{St}(\alpha))$.}

Lemma 3.1. Let $\varphi(z)$ be a finite Blaschke product with $\varphi(0)=0$ and let $c$ be a complex number with $|c|=1$. If $\alpha<1 / 2$ and $\varphi(z) /(1-c \varphi(z))^{2(1-\alpha)}$ is a support point of $S(\operatorname{St}(\alpha))$ then $\varphi(z)=x z$ for some $|x|=1$.

Proof. We first note that a result in $[6$, p. 83] gives

(*) $\frac{1+c \varphi(z)}{1-c \varphi(z)}=\sum_{k=1}^{n} \lambda_{k} \frac{1+x_{k} z}{1-x_{k} z}$ where $n$ is a positive integer,

$$
\left|x_{k}\right|=1, \lambda_{k}>0(k=1,2, \ldots, n) \text { and } \sum_{k=1}^{n} \lambda_{k}=1 .
$$

If we let $q=1-2 \alpha(>0)$, then

$$
\begin{aligned}
& \frac{\varphi(z)}{(1-c \varphi(z))^{2(1-\alpha)}}=\bar{c}\left\{\frac{c \varphi(z)}{1-c \varphi(z)}\right\} \cdot\left\{\frac{1}{1-c \varphi(z)}\right\}^{q} \\
& =\sum_{k=1}^{n} \lambda_{k} \frac{\bar{c} x_{k} z}{1-x_{k} z} \cdot h(z) \text { where } h(z)=\left\{\frac{1}{1-c \varphi(z)}\right\}^{q}
\end{aligned}
$$

and we have used $(*)$ in the second equality. By Lemma 1.1 we have

$$
\begin{gathered}
\frac{x_{k} z}{1-x_{k} z} h(z)=\int \frac{x z}{(1-y z)^{2(1-\alpha)}} d \lambda(x, y), \text { and thus } \\
\frac{\bar{c} x_{k} z}{1-x_{k} z} h(z)=\int \frac{x z}{(1-y z)^{2(1-\alpha)}} d \lambda_{1}(x, y) .
\end{gathered}
$$


By Theorem 1.2, $\bar{c} x_{k} z /\left(1-x_{k} z\right) h(z)$ belongs to $\overline{\operatorname{co}} S(\operatorname{St}(\alpha))$. Consequently if

$$
\begin{aligned}
\frac{\varphi}{(1-c \varphi)^{2(1-\alpha)}} & =\left\{\sum_{k=1}^{n} \lambda_{k} \frac{\bar{c} x_{k} z}{1-x_{k} z}\right\} h(z) \\
& =\sum_{k=1}^{n} \lambda_{k}\left\{\frac{\bar{c} x_{k} z}{1-x_{k} z} h(z)\right\}
\end{aligned}
$$

is a support point of $S(\operatorname{St}(\alpha))$, hence also of $\overline{\operatorname{co}} S(\operatorname{St}(\alpha))$, then so is each term. That is, $\left(\bar{c} x_{k} z /\left(1-x_{k} z\right)\right) h(z)$ is a support point of $\overline{\operatorname{co}} S(\operatorname{St}(\alpha))$.

Now by Theorem 2.2 we must have

$$
\left\{\frac{x_{k} \bar{c} z}{1-x_{k} z}\right\} \cdot h(z)=\int_{U} \frac{\overline{B_{k}(y)} z}{(1-y z)^{2(1-\alpha)}} d \nu_{k}(y)
$$

for some finite Blaschke product $B_{k}(z)$ and some probability measure $\nu_{k}$ on $U(k=1,2, \ldots, n)$. In view of $(*)$ we can write this as

$$
\left\{\frac{x_{k} \bar{c} z}{1-x_{k} z}\right\} \cdot\left\{\sum_{J=1}^{n} \lambda_{J} \frac{1}{1-x_{j} z}\right\}^{q}=\int_{U} \frac{\overline{B_{k}(y)} z}{(1-y z)^{2(1-\alpha)}} d \nu_{k}(y) .
$$

Comparison of the $z$ coefficient of both sides yields

$$
\int_{U} \overline{B_{k}(y)} d \nu_{k}(y)=x_{k} \bar{c}
$$

which implies that $\nu_{k}$ is a point mass at some $w_{k}\left(\left|w_{k}\right|=1, k=1,2, \ldots, n\right)$. Hence we have

$$
\begin{gathered}
\left\{\frac{x_{k} \bar{c} z}{1-x_{k} z}\right\} \cdot\left\{\sum_{j=1}^{n} \lambda_{J} \frac{1}{1-x_{j} z}\right\}^{q}=\frac{\overline{B_{k}\left(w_{k}\right)} z}{\left(1-w_{k} z\right)^{2(1-\alpha)}} \quad \text { and } \\
\overline{B_{k}\left(w_{k}\right)}=x_{k} \bar{c} .
\end{gathered}
$$

Now since $q=1-2 \alpha>0(\alpha<1 / 2)$, comparison of singularities of the above equation gives $n=1$, as required.

THEOREM 3.2. Let $\alpha<1 / 2$ and $J$ be a continuous linear functional on $\mathscr{A}$ not of the form $J(f)=a f(0)+b f^{\prime}(0)(a, b \in \mathbf{C}$ and $f \in \mathscr{A})$. If $f_{0}$ is a support point of $S(\operatorname{St}(\alpha))$ associated with $J$, then $f_{0}(z)=x z /(1-y z)^{2(1-\alpha)}$.

Proof. Let $f_{0} \prec g_{0}$ where $\left.g_{0} \in \operatorname{St}(\alpha)\right)$ and consider $\mathscr{G}=\{f \in \mathscr{A} \mid f \prec$ $\left.g_{0}\right\}$. Then $f_{0}$ is in $\mathscr{G}$ and $\operatorname{Re} J$ peaks over $\mathscr{G}$ at $f_{0}$. If $\operatorname{Re} J$ is constant over $\mathscr{G}$ then $\operatorname{Re} J\left(g_{0}\left(x z^{m}\right)\right)=$ constant, for all $|x|=1, m=1,2,3, \ldots$ Hence $J\left\{z^{m}\right\}=0(m=1,2, \ldots)$, which violates the assumption on the form on 
$J$. Thus $\operatorname{Re} J$ is non constant over $\mathscr{G}$ and $f_{0}$ is a support point of $\mathscr{G}$. By a result of Abu-Muhanna [1], (see also [10]), $f_{0}(z)=g_{0}\left(\varphi_{0}(z)\right.$ ) where $\varphi_{0}$ is a finite Blaschke product with $\varphi_{0}(0)=0$. We claim $\varphi_{0}(z)=x_{0} z$ for some $\left|x_{0}\right|=1$ and $g_{0}(z)=z /(1-c z)^{2(1-\alpha)}$ for some $|c|=1$. To see this define $L$ on $\operatorname{St}(\alpha)$ by $L(g)=J\left\{g\left(\varphi_{0}(z)\right)\right\}$. Then $L$ is a continuous linear functional on $\mathscr{A}$ and $\operatorname{Re} L$ peaks over $\operatorname{St}(\alpha)$ at $g_{0}$. If $\operatorname{Re} L$ is non constant over $\operatorname{St}(\alpha)$ then $g_{0}$ becomes a support point of $\operatorname{St}(\alpha)$, and $g_{0}(z)=$ $z /(1-c z)^{2(1-\alpha)}$ for some $|c|=1[6, \mathrm{p} .89]$.

Hence $f_{0}(z)=\varphi_{0(z)} /\left(1-c \varphi_{0}(z)\right)^{2(1-\alpha)}$ and, by Lemma 3.1, $\varphi_{0}(z)=$ $x_{0} z$ with $\left|x_{0}\right|=1$ as desired. If $\operatorname{Re} L$ is constant over $\operatorname{St}(\alpha)$, then $\operatorname{Re} J\left\{g\left(\varphi_{0}(z)\right)\right\}=\operatorname{Re} J\left\{g_{0}\left(\varphi_{0}(z)\right)\right\}$ for all $g$ in $\operatorname{St}(\alpha)$, and hence $g\left(\varphi_{0}(z)\right)$ is a support point of $S(\operatorname{St}(\alpha))$ for all $g$ in $\operatorname{St}(\alpha)$. In particular this is true when $g(z)=z /(1-c z)^{2(1-\alpha)}$ and so $\varphi_{0}(z) /\left(1-c \varphi_{0}(z)\right)^{2(1-\alpha)}$ is a support point of $S(\operatorname{St}(\alpha))$. Again, by Lemma 3.1, $\varphi_{0}(z)=x_{0} z$ for some $\left|x_{0}\right|=1$. We now have $\operatorname{Re} J\left\{g\left(x_{0} z\right)\right\}=$ constant, for all $g$ in $\operatorname{St}(\alpha)$. If we take $g(z)=z /(1-x z)^{2(1-\alpha)},|x|=1$, it follows that $J\left(z^{n}\right)=0, n=2$, $3, \ldots$, again violating the assumed form of $J$. Consequently $\operatorname{Re} L$ is non constant over $\operatorname{St}(\alpha)$ and the theorem follows.

ReMarKs. (1) It is not difficult to show that each function

$$
x z /(1-y z)^{2(1-\alpha)} \quad(|x|=|y|=1)
$$

is a support point corresponding to a continuous linear functional $J$ not of the form $J(f)=a f(0)+b f^{\prime}(0)$.

(2) Theorem 3.2 is not true for $1 / 2 \leq \alpha<1$. For example

$$
z^{n} /\left(1-x z^{n}\right) \quad(|x|=1, n=1,2, \ldots)
$$

is always a support point of $S(\operatorname{St}(\alpha))$ when $1 / 2 \leq \alpha<1$. Moreover, if $\alpha=1 / 2$, with a trivial modification of the proof given in [10] for $\Sigma S(K)$, where $K$ is the usual subclass of convex functions, one can show that

$$
\sum\left(S\left(\operatorname{St}\left(\frac{1}{2}\right)\right)\right)=\left\{f \circ \varphi \mid f \in \operatorname{St}\left(\frac{1}{2}\right) \text { and } \varphi\right.
$$

$$
\text { is a finite Blaschke product with } \varphi(0)=0\} \text {. }
$$

Acknowledgment. We wish to thank Professor T. H. MacGregor for helpful conversations.

\section{REFERENCES}

[1] Y. Abu-Muhanna, Variability region and support points of subordinate families, to appear.

[2] D. A. Brannan, J. G. Clunie and W. E. Kirwan, On the coefficient problem for functions of bounded boundary rotations, Ann. Acad. Sci. Fenn. Ser. A VI Phys., 523 (1973), 18 pp. 
[3] L. Brickman, D. J. Hallenbeck, T. H. MacGregor and D. R. Wilken, Convex hulls and extreme points of families of starlike and convex mappings, Trans. Amer. Math. Soc., 185 (1973), 413-428.

[4] L. Brickman, T. H. MacGregor and D. R. Wilken, Convex hulls of some classical families of univalent functions, Trans. Amer. Math. Soc., 156 (1971), 91-107.

[5] D. G. Cantor and R. R. Phelps, An elementary interpolation theorem, Proc. Amer. Math. Soc., 16 (1965), 523-525.

[6] P. C. Cochrane and T. H. MacGregor, Fréchet differentiable functionals and support points for families of analytic functions, Trans. Amer. Math. Soc., 236 (1978), 75-92.

[7] E. Grassman, W. Hengartner and G. Schober, Support points of the class of close to convex functions, Canad. Math. Bull., 19, \#2 (1976), 177-179.

[8] D. J. Hallenbeck, Extreme points of classes of functions defined through subordination, Proc. Amer. Math. Soc., 46 (1) (1974), 59-64.

[9] D. J. Hallenbeck and T. H. MacGregor, Subordination and extreme point theory, Pacific J. Math., 50 (1974), 455-468.

[10] _ Support points of families of analytic functions described by subordinations, Trans. Amer. Math. Soc., 278 (2) (1983), 523-546.

[11] T. H. MacGregor, Applications of extreme point theory to univalent functions, Michigan Math. J., 19 (1972), 361-376.

[12] A. A. S. Perera, Support points and extreme points of some classes of analytic functions, Doctoral Dissertation, State University of New York at Albany, 1983.

[13] W. Rogosinski, On the coefficients of subordinate functions, Proc. London Math. Soc., (2) 48 (1943), 48-52.

Received December 10, 1984.

SUNY

AlbANY, NY 12203 



\section{PACIFIC JOURNAL OF MATHEMATICS EDITORS}

V. S. VARADARAJAN

(Managing Editor)

University of California

Los Angeles, CA 90024

HERBERT CLEMENS

University of Utah

Salt Lake City, UT 84112

R. FINN

Stanford University

Stanford, CA 94305
HERMANN FLASCHKA

University of Arizona

Tucson, AZ 85721

RAMESH A. GANGOLLI

University of Washington

Seattle, WA 98195

VAUGHAN F. R. JONES

University of California

Berkeley, CA 94720

ROBION KIRBY

University of California

Berkeley, CA 94720
C. C. MOORE

University of California

Berkeley, CA 94720

H. SAMELSON

Stanford University

Stanford, CA 94305

HAROLD STARK

University of California, San Diego

La Jolla, CA 92093

\section{ASSOCIATE EDITORS}

R. ARENS

E. F. BECKENBACH

B. H. NEUMANN (1906-1982)
F. WOLF

K. YOSHIDA

\section{SUPPORTING INSTITUTIONS}

UNIVERSITY OF ARIZONA

UNIVERSITY OF BRITISH COLUMBIA

CALIFORNIA INSTITUTE OF TECHNOLOGY

UNIVERSITY OF CALIFORNIA

MONTANA STATE UNIVERSITY

UNIVERSITY OF NEVADA, RENO

NEW MEXICO STATE UNIVERSITY

OREGON STATE UNIVERSITY
UNIVERSITY OF OREGON UNIVERSITY OF SOUTHERN CALIFORNIA

STANFORD UNIVERSITY

UNIVERSITY OF HAWAII

UNIVERSITY OF TOKYO

UNIVERSITY OF UTAH

WASHINGTON STATE UNIVERSITY

UNIVERSITY OF WASHINGTON 


\section{Pacific Journal of Mathematics}

\section{Vol. 123, No. $1 \quad$ March, 1986}

Maria Emilia Alonso García, A note on orderings on algebraic varieties $\ldots \ldots 1$

F. S. De Blasi and Józef Myjak, On continuous approximations for

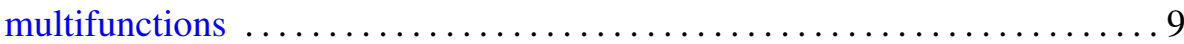

Frank Albert Farris, An intrinsic construction of Fefferman's CR metric . . . 33 Antonio Giambruno, P. Misso and Francisco César Polcino Milies, Derivations with invertible values in rings with involution $\ldots . \ldots . \ldots .47$

Dan Haran and Moshe Jarden, The absolute Galois group of a pseudo real

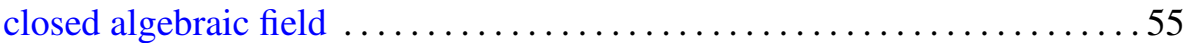

Telemachos E. Hatziafratis, Integral representation formulas on analytic

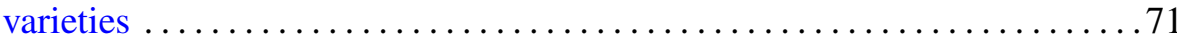

Douglas Austin Hensley, Dirichlet's theorem for the ring of polynomials

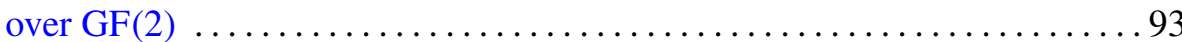

Sofia Kalpazidou, On a problem of Gauss-Kuzmin type for continued

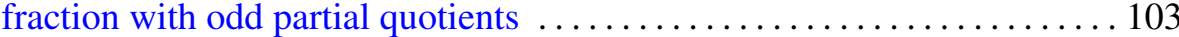

Harvey Bayard Keynes and Mahesh Nerurkar, Ergodicity in affine

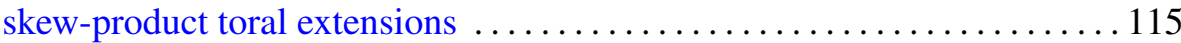

Thomas Landes, Normal structure and the sum-property $\ldots \ldots \ldots \ldots \ldots 127$

Anthony To-Ming Lau and Viktor Losert, Weak*-closed complemented invariant subspaces of $L_{\infty}(G)$ and amenable locally compact groups ...149 Andrew Lelek, Continua of constant distances in span theory . . . . . . . . 161 Dominikus Noll, Sums and products of $B_{r}$ spaces $\ldots \ldots \ldots \ldots \ldots \ldots \ldots \ldots$ Lucimar Nova, Fixed point theorems for some discontinuous operators 189

A. A. S. Perera and Donald Rayl Wilken, On extreme points and support points of the family of starlike functions of order $\alpha$

Massimo A. Picardello, Positive definite functions and $L^{p}$ convolution operators on amalgams ........................

Friedrich Roesler, Squarefree integers in nonlinear sequences ......... 223

Theodore Shifrin, The osculatory behavior of surfaces in $\mathbf{P}^{5}$ 